\title{
Review of The Future Academic
}

\section{Librarian's Toolkit: Finding Success on the Job Hunt and in Your First Job}

Rachel Evans

University of Georgia

Abstract: Review of Hodge, Megan (Ed.) (2019). The Future Academic Librarian's Toolkit:

Finding Success on the Job Hunt and in Your First Job. Chicago: Association of College and Research Libraries.

Keywords: academic libraries, academic librarian, job satisfaction, job hunt, academia

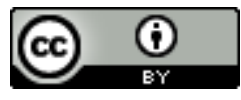

This is an Open Access article distributed under the terms of the Creative Commons Attribution 4.0 International License (http://creativecommons.org/licenses/by/4.0), which permits unrestricted use, distribution, and reproduction in any medium, provided the original work is properly cited. 
The world of librarianship is a weird and wild path. It is also wonderful, partly because of how diverse of a career it can be. What attracts people to the arena of libraries is often their inquisitive spirit. Their passion for lifelong learning, the enjoyment they find in acquiring generalist knowledge, and their relative ease for absorbing new skills make them ideal chameleons when challenges arise. The Future Academic Librarian's Toolkitembraces these characteristics in the new librarian and expands on the wide palette that is academic librarianship. It reads both like a textbook, complete with definitions and summaries of this type of career, and like a self-help guide, with practical tips and advice, templates, tables and appendices which may be copied and used repeatedly or filled in workbook-style. The breakdown of parts, chapters, and the layout of the content make this otherwise dry subject matter easy and enjoyable to take in. Reading it truly is a pleasure.

One beneficial aspect of the book is the bibliography closing out each section of text. I found myself returning to favorite sentences and using that bibliography to find a more extensive article or book on the topic. There were several areas that truly inspired confidence in me or invigorated me with an idea or a different approach. A helpful table early in the text "Telling your Story: Leveraging Transferable Skills" is a great example of how applicable the content is in a variety of contexts. As a primarily visual learner I love seeing skills laid out and categorized the way this table does (p. 78-79). From the beginning, it sets the tone for illustrating how many different flavors of people, skills, educational and economic backgrounds can find happiness and success in librarianship.

Another favorite section was Chapter 5: Finding a Mentor (p.107-108) and Growing Pains (p. 108-110). Excellent advice is pooled together from several trusted sources and paired 
perfectly with bulleted tips and additional resources. Mentors are not always obvious upon first glance and trying to formally identify a single individual can sometimes lead to missed informal opportunities for mentorship that may be more valuable long term. The handful of paragraphs around the 100-page mark helped me to open my eyes and notice some more organic relationships that I had not yet realized had a mentoring dynamic.

Of course, not everything in this book is for everyone. For me in particular, Liaison Librarianship (p.115-132) was one such chapter. There were, of course, gems of truth, expertise, and advice in Chapter 6, but the liaison-model is not found in every academic library. In this way, other chapters may or may not apply to every academic librarian depending on your organizational structure, management styles, and size. Still, it was valuable for gaining an understanding of how other institutions work.

Chapter 8 was by far one of the most interesting sections to read in entirety. In Academic Librarian Roles Beyond Public Services (p. 153-189), you are introduced specifically to five different job titles found in many academic libraries. More variations are shared, the scope of actual tasks, specific skills, and other aspects of a hypothetical librarian's profile are laid out and dissected. The overlap of traditionally ubiquitous departments found in libraries is brought to the forefront in the chapter introduction. The intertwining nature of technical services, metadata, electronic resources, web services, systems, communication, data management, digital scholarship, and more is highlighted (p. 153). The best part of each job overview is the "A Day in the Life" portion, which makes an excellent effort to capture and explain each position's possible expectations. 
For Job 2: Academic Data Librarian, another exemplary visual is provided - titled "Librarian Roles in the Research Lifecycle" (Figure 8.2, p.166). Additionally, the organizations, professional publications, and seminal texts outlined in Job 4: Special Collections and Archives Librarian section provides great starting points for new and established professionals alike (p.179-180). I would have liked to see even more a variety of job profiles and would recommend an expanded set laid out in a similar fashion to provide further guidance for new or transitioning librarians.

Chapters 9 through 13 (p. 191-312) all contain wonderful examples of leadership skills. Much of this content centered around preparing for and planning your future career, long term impact, and even potential academic reputation. Topics include obvious networking and conference advice, as well as not so obvious negotiation and persuasion tactics for navigating the politics of academia. These chapters left me feeling more empowered. I found myself taking notes during these later portions of the book and actively applying them in the workplace right away. Advocating for yourself is a difficult process and finding your niche in any profession is no small feat either. Identifying areas of opportunity, gaining awareness of your own weaknesses, and learning to leverage your strengths in a work setting is something surely everyone can utilize, no matter how many years one does or does not have invested in any given role.

"Plan Your Impact" (Chapter 12, p.265-288) is another standout section I appreciated on multiple levels. Priceless advice encourages, even from the chapter subtitle, to “... make yourself irreplaceable". Here it defines success very effectively by summarizing expectations for progression in our modern shape-shifting industry of information: "Succeeding as an academic 
librarian requires the construction and maintenance of a professional reputation, involvement in professional organizations, and construction of effective narratives ... for promotion ..." (p. 265). The text recommends smartly stacking your skills for establishing job security and extended opportunities for increased leadership and the respect of peers. This process begins, as advised in this chapter, by creating your own solid, measurable goals and aligning them with the organization you are either in or wish to be a part of.

Along this line of thought, the remainder of this chapter strategically defines mindsets, provides a checklist of questions to ask yourself about other professionals you admire, and even delves into the vast and confusing landscape of crafting your career-based social media presence. The online persona segments encourage readers to find their own way with their professional identities and consciously set comfortable limits based on research and observation. It addresses top-level decisions like anonymity and privacy levels and concerns for various platforms and also microcosm choices with example Twitter and blog handles, profile images, and more. Towards the end, a great little weekly schedule is provided for getting started with a professional social media presence (p.275) and professional website (p.276). Further on, tips for assessing your progress and maximizing your impact are provided in numerical lists varied by example types that begin with work-related project sequencing. This chapter includes a very handy table (12.3 Online Persona Development Options, p. 285) for quickly identifying and comparing the pros and cons of several popular online spaces.

In conclusion, this toolkit is highly recommended. Every aspiring academic librarian could benefit from the wisdom within. For current librarians in any field, the content is not only useful for those entering or changing positions but seems primed as a ready reference item for 
all workplaces dealing with any of the subjects addressed. A valuable contribution to the professional development collection of my own office, and one I will surely share with colleagues for years to come. 